\title{
SEMANTIC AND PRAGMATIC INTERPRETATION OF JAPANESE SENTENCES WITH DAKE (ONLY)
}

\author{
NAOIIKO NOGUCHI* YASUNARI HARADA ${ }^{\dagger}$
}

\author{
CSLI \\ Ventura IIall, Stanford University \\ Stanford, CA 94305-4115, U.S.A.
}

\section{Introduction}

Separating semantics and pragmatics is important for the design of natural language systems, as well as for linguistic purposes, because the former is inlierently dependent on particular lexical items involved or the overall organization of the particular language in question, thus comprising a language-dependent part of the interpretive system, whereas the latter is essentially related to a more general and presumably languageindependent reasoning processes of the human or other agents involved in dialogues.

Previous linguistic study or the semantics of Japanese functional words such as dake did not pay enough attention to carefully distinguishing the pragmatic factors involved from the (lexical) semantic contents of those words. In order to build efficient natural language systems, however, we believe that not only do we have to account for the semantics of each and every lexical items under consideration, but we need to have a general account of certain pragmatic aspects of the interpretations we obtain.

One typical case of this kind of pragmatic inferences manifests itself in interpretations of Japanese sentences with $d_{a k e}$, which roughly corresponds to the English word only. We believe that the kind of analysis we propose here is a prototypical example of what is necessary for successful natural language interpretation.

\section{Differences in Interpretation of Dake-sentences}

It has been observed that there is a certain difference in meaning between the Japanese sentences shown in $(1, a)$ and $(1, b)$, according to the relative positioning of the two particles, dake (only) and de (by). (We will call the first type of sentences de-dake-sentences and the second type dake-de-sentences for short. We will also call them in general, dake-sentences.)

(1) a. de-dake-sentence:

"also at Matsushita Electric Industrial, Co., Ltd.

talso at Waseda Univerbity
Soko-ni-wa zitensya de dake ik-eru. there-rOC-TOP bike INST only go-can ((I) can get there only by bike.)

b. dake-de-sentence:

Soko-ni-wa zitensya dake de ik-eru. there-LOC-Top bike only INST go-can ((I) can get there by bike alone.)

According to Morita[8], $(1, a)$ means that "bike is the only means by which 1 can get there," i.e. "I can't get there by any neans other than bike." He called this the 'absolute restriction' meaning. On the other hand, $(1, \mathrm{~b})$ roughly means that "I can get there by bike alone," i.e. "the minimally necessary means which enables me to get there is by bike." This he called the "minimal restriction' meaning. In this case, we have a reading in which "I can get there by any other means easier than bike."

We can sec that there is a similar difference in available readings for the corresponding English sentences, which are shown in (2). While $(2, a)$ has the 'absolute restriction' meaning, $(2, b)$ has the 'minimal restriction' meaning, in Morita's terminology.

(2) a. I can get there only with a bike.

b. I can get there with only a bike.

It might be suggested that the difference in the meanings of these sentences are due to the relative positioning of dake and de, in the case of Japanese, and that of only and with, in the case of English, which somehow causes the difference in the semantic scopes of dake or only. But when we look at other examples like (3), in which dake interact with particles other than de, it becomes obvious that the real phenomenon is a bit more complicated.

(3) i. Sono-koto-wa haha ni dake i-eru. that-thing-ToP mother DAT only tell-can ((I) can tell it only to my mother.)

b. Sono-kotowa haha dake ni i-eru. that-thing-TOP mother only DAT tell-can ((I) can tell it to only my mother.)

In (3), we see that $n i$ can either precede or follow dake, as in the case of $d e$. However, the difference in 
meaning between the two sentences in (3) is not at all clear. This is also true of the English equivalents given above. Both sentences mean that "I can't tell it to any person other than my mother."

These sentences seem to suggest that there is something common to Japanese and English, an interpretive procedure for sentences involving dake and only, which effects in some difference in meaning in the case of pairs of sentences in (1) or in (2), but does not in the case of pairs of sentences in (3).

In this paper, we will focus on these sentences and formulate the interpretive procedure which would explain the differences in these interpretations.

\section{Previous \\ Accounts \\ for Japanese}

The difference in interpretation between de-dakesentences and dake-de-sentences has attracted attentions of Japanese linguists. We will summarize here Morita's[8] observations and Kuno's[5] generalizations.

Morita[8] was the first to observe this difference and characterized these sentences as follows.

Morita's observations:

(4) a. De-dake means 'absolute (exclusive) restriction'.

b. Dake-de means 'minimal restriction'.

c. The alternation between dake and particles other than de doesn't ca use this kind of difference. Each ordering means 'absolute restriction'.

Kuno[5] generalized Morita's observations to the ordering of particles in general and ascribed the difference in meaning of de-dake-sentences and dake-de-sentences to the semantic contents of these particles and the order they appear in the sentence. His generalizations can be summarized as in (5).

Kuno's generalizations:

(5) a. The ordering of particles (particle + quantifierlike particl e), such as de-dake, ni-dake, to-dake, de-nomi, de-bakari, etc. means 'absolute (exclusive) restriction'.

b. The ordering of particles (quantifier-like particle + particl e) such as dake-de, dake-ni, daketo, nomi-de, bakari-de, etc. means 'minimal restriction'.

c. The alternation between dake and particles other than de does cause the difference in meaning, but in some cases, the ordering (quantifierlike particle + particle) have 'absolute restriction' meaning as a secondary meaning.

From now on, we will call the ordering of particles (particle + dake), as in de-dake, p(article)-dake, the ordering (dake + particle〉, dake-p(article). And we will also call sentences with these orderings p-dakesentences and dake-p-sentences, respectively.
One common feature of these two accounts is that they are trying to capture the difference between the two types of sentences in the semantic contents of particular orderings of particles. On that basis, Morita claims that the semantic contents of de-dake and dakede are different while relative order of dake and particles other than de does not affect the semantic contents of these sentences. Kuno, on the other hand, claims that the semantic contents of $p$-dake and dake-p are always different, and dake- $p$ has one additional reading in some cases, in which it is equivalent to that of $p$-dake.

However, sentences like (6) is a straight-forward counterexample to their claims. Here, a dake-de sentence does not have the 'minimal restriction' meaning, although what Kuno meant exactly by 'the minimal restriction meaning' is somewhat unclear.

(6) Soko-ni-wa zitensya dake de i-tta. there-LOC-TOP bike only INST go-PAST ((I) got there by bike alone.)

It is clear that the difference in meaning between $p$ dake-sentences and dake-p-sentences is a difference in the interpretations of the whole of these sentences, not a difference in the semantic contents of the particular part of the sentences.

In what follows, we will take another careful look at the details of the so-called 'minimal restriction' meaning of dake-p-sentences and try to figure out what kind of interpretive procedure is involved in causing these differences.

\section{Another Look at the Differ- ences in Interpretation}

\subsection{The 'Minimal Restriction' Meaning as a Composite}

If we look at the original dake-de-sentence $(1, \mathrm{~b})$ carefully, the intuitive interpretation we obtain is something like "bike provides a sufficient means for getting there, and any other means is not necessary." Moreover we feel that "I can get there by any other means easier (in some sense) than bike."

Thus, what Kuno and Morita called 'minimal restriction' meaning can be reformulated in terms of the following two statements in ( 7 ).

'Minimal restriction' meaning:

(7) a. Anything other than $X$ is not necessary. ("necessity' part)

b. Anything "bigger" or "more costly" than $X$ will suffice. ('scalar' part)

In the case of $p$-dake sentences, we see that the 'absolute restriction' meaning is a part of its semantic content. For instance, in $(8)$, we cannot utter $(8, \mathrm{~b})$ after uttering $(8, a),(8, b)$ is incompatible with the 'absolute restriction' meaning of $(8, a)$. This shows that the ' $a b$ solute restriction' meaning of p-dake-sentences is not 
defeasible, so it is a part of semantic contents of dedake-sentences.

(8) a. Soko-ni-wa zitensya de dake ik-eru. there-Loc-Top bike INST only go-can ((I) can get there only by bike.)

b. ??Zidoosya de mo ik-eru.

$$
\begin{aligned}
& \text { car INST too go-can } \\
& \text { ((I) can get (there) by car, too.) }
\end{aligned}
$$

But how about the 'minimal restriction' meaning of dake-p-sentences? A similar test can be applied. In the following examples, uttering $(10, \mathrm{~b})$ after uttering $(9)$ is weird, whereas uttering $(10, a)$ or $(10, c)$ after $(9)$ is not strange at all.

(9) Sokoni-wa zitensya dake de ik-eru. there-LOC-TOPbike only INST go-can ((I) can get there by bike alone.)

(10) a. Zidoosya de mo ik-eru. car INST too go-can ((I) can get (there) by car, too.)

b. ??Zidoosya tno hituyoo-da. car too necessary-is (A car is necessary, too.)

c. Zidoosya de wa ik-e-nai. car INST TOP go-can-not ((I) can't get (there) by car.)

This shows that the 'necessity' part of the 'minimal restriction' meaning of dake-p-sentences is not defcasible, and is an inherent part of the semantic content, whereas the 'scalar part' is defeasible, a kind of conversational implicature.

Given these observations, we propose the following hypotheses in order to explain the differences in interpretation between $p_{u} d a k e-s e n t e n c e s$ and dake-psentences.

\section{Hypotheses:}

(A) While p-dake-sentences always have the 'absolute restriction' meaning as a part of their semantic contents, dake-p-sentences do not have it in some cases. (These two types of sentences have a difference in their semantics in this respect.)

(B) Dake-p sentences have the meaning that anything other than the thing in question is not necessary ('necessity' part of the 'minimal restriction' meaning), as a part of their semantic contents, depending on their contexts.

(C) We can get the 'scalar' part of the 'minimal restriction' meaning from dake-p-gentences in some contexts.

(D) This meaning of dake-p-sentences can be seen as a kind of conversational implicature obtained through some praginatic inference of the hearer.
(A) and (B) above are concerned with the semantics, while (C) and (D) are concerned with the pragmatics. In the remainder of this paper, we will concentrate on Japanese examples, but we believe that a similar if not identical, processes are involved in the interpretation of English counterparts.

\subsection{Further Observations}

Given the hypotheses (A)-(D) that explains the differences in available readings between $p$-dake-sentencey and dake-p-sentences, the following questions have to be raised.

- With regard to $(\mathrm{A})$ and $(\mathrm{B})$, what are the contexts where dake-p-sentences do not have 'absolute restriction' meaning, and in what contexts do they have the 'necessity' part as their semantic contents, and how?

- With regard to (C) and (D), in what contexts do dake-p-sentences get the 'scalar' part, and how?

In (6) we saw an example where dake-p-sentences do not have the 'minimal restriction' meaning. In other words, (6) have neither the 'necessity' part nor the 'scalar' part. Here are some other examples.

$$
\begin{aligned}
& \text { a. Sokoni-wa zitengya de dake } \\
& \text { there-Tor bike INST only } \\
& \text { i-tta-koto-ga-aru. } \\
& \text { go-PAST-that-Nom-exist } \\
& \text { ((I) have been there only by bike.) } \\
& \text { b. Sokoni-wa zitensya dake de } \\
& \text { there-TOY bike only INST } \\
& \text { i-tta-koto-ga-aru. } \\
& \text { go-PAST-that-Nom-exist } \\
& \text { ((I) have been there by bike alone.) }
\end{aligned}
$$

$(11, a)$ means that "I haven't been there by any means other than bike," that is, it has the 'absolute restriction' meaning, and $(11, b)$ clearly does not have the 'absolute restriction' meaning. Although this difference in meaning between these two sentences is clear, $(11, \mathrm{~b})$ does not have the 'minimal restriction' meaning. That is, this sentence have neither the 'necessity part' nor the 'scalar part' of the 'minimal restriction' meaning.

But there are other examples in which we can get the 'necessity part' and 'scalar part' of the 'minimal restriction' meaning, as in (12).

(12) a. Kotosi-no kaze-wa tyuusya de dake this-year-of cold-TOP injection INST only naoru.

calt-be-cured

(This year's cold can be cured only by injection.)

b. Kotosi-no kaze-wa tyuusya dake de this-year-of cold-Top injection only INST naoru. 
can-be-cured

(This year's cold can be cured by injection alone.)

Even among sentences involving dake and $d e$, there are differences in available interpretations. The only difference among these sentences lies in the properties of their predicates. We see that the 'minimal restriction' meaning for dake-p-sentences is obtained only when the predicates involved express some "possibility," such as ik-eru (can go) or naoru (can be cured).

Taking into account examples that involve dake along with particles other than de, we notice that things get further complicated. We have already seen in (3) that in sentences involving dake and $n i$, we can get neither the 'necessity' part nor the 'scalar' part of the 'minimal restriction' meaning for a dake-ni-sentence, even when we use a "possible" predicate. When we do not use a "possible" predicate, we can't get the 'minimal restriction' meaning for dake-ni-sentences either, as shown in (13).

(13) a. Kazoku ni dake siraseru.

family DAT only inform

((I) will inform (it) only to my family.)

b. Kazoku dake ni siraseru.

family only DAT inform

(I) will inform (it) to only my family.)

From these observations, we have at least partial answers to the two questions we raised at the beginning of this section. As for the first question, we can say that when dake-p-sentences involve certain predicates like 'ikeru', 'itta-koto-ga-aru', the 'absolute restriction' meaning tends to disappear. Moreover, when they involve de and "possible" predicates, they have the "necessity' part as their semantic contents. For the second question, we would say that when dake-p-sentences involve de and "possible" predicates, they also have the 'scalar' part as their conversational implicature. When they involve $d e$ but not "possible" predicates, they neither have the 'necessity' part nor the 'scalar' part of the 'minimal restriction' meaning.

These answers suggest that each part of the 'minimal restriction' meaning is tightly related, and, the particle de and "possible" predicates both play crucial roles in this phenomenon.

\section{The Interpretive Procedure for Dake-sentences}

\subsection{De and "Possible" Context}

In the previous section, we observed that de-phrases and "possible" predicates are crucial to the difference in meaning between p-dake-sentences and dakep-sentences. Then what is going on when de-phrases and "possible" predicates interact with each other?

In order to see this, first consider what happens when we omit dake from (1) as shown in (14).
(14) Sokoni-wa zitensya de ik-eru. there-TOP bike INST go-can ((I) can get (there) by bike.)

Intuitively, this sentence can be paraphrased as (15), without any difference in its interpretation.

(15) Sokoni-wa zitensya-o tukae-ba

there-Top bike-ACC use-if

iku-koto-ga-dekiru.

go-that-NOM-can

(If (I) use a bike, (I) can get there.)

We can get this conditional interpretation only when the sentence has a "possible" predicate as well as a de-phrase. As sentences in (16) and (17) show, this kind of interpretation for sentences with a de-phrase is unavailable when we do not have a "possible" predicate.

(16) Zitensya de iki-taj.

bike INST go-want

((I) want to go (there) by bike.)

(17) Zitensya de iku-bekida.

bike INST go-should

((You) should go (there) by bike.)

Similarly, if there is no de-phrase, we do not get the conditional interpretation even when we have a "possible" predicate, as the following examples demonstrate.

(18) Zitensya o ka-eru.

bike ACC buy-can

((I) can buy a bike.)

(19) Tookyo kara okur-eru.

Tokyo fromsend-can

((I) can send (it) from Tokyo.)

(20) Taroo ni a-eru.

Taroo DAT meet-can

((I) can meet with Taroo.)

When de-phrases and "possible" predicates interact, the conditional interpretation becomes available. Note, incidentally, that in English too, similar observations can be made. Consider the English equivalent to (14), shown in (21). This sentence can be interpreted as synonymous with a conditional sentence in (22).

(21) I can get there with a bike.

(22) If I use a bike, I can get there.

Stump[10] discusses this kind of interaction between "possible" predicates and free adjuncts. His main concern is how free adjuncts behave in modal contexts, and the typical examples he considers are shown in (23) and (24).

(23) a. Wearing that new outfit, Bill would fool everyone.

b. If he wore that new outfit, Bill would fool everyone.

(24) a. Standing on a chair, John can touch the ceiling. 
b. If lie stands on a chair, John can touch the ceiling.

According to his observations, $(23, \mathrm{a})$ and $(24, \mathrm{a})$ are interpreted as their corresponding conditional sentences in $(23, b)$ and $(24, b)$. Itis proposal is that this kind of conditional interpretation for free adjuncts becornes available within semantics of modals. Free adjuncts would be assinged the same semantic type as conditional clauses, and so the conditional interpretation derives entirely from the semantic rules necessary for the interpretation of modals.

He uses Kratzer's[3] formalizalion for the semantic rules for modals; the semantic contents of (23) and (24) are expressed as follows.

(25) would $\left(\mathrm{D}(\mathrm{cb})\left({ }^{\sim}\right.\right.$ Bill_wears_that_new_outfit’ $\left.)\right)$ ( ${ }^{\wedge}$ Bill_fools_everyone')

(26) $\operatorname{can}^{\prime}\left(\mathrm{D}(\mathrm{cb})\left({ }^{-}\right.\right.$John_stands_on_a_chair' $\left.)\right)$

("John_touches_the_ceiling')

Kratzer uses a possible world semantics, and a simple way to read these formulae is to understand that $\mathrm{D}$ is some function which maps the conversational background, $\mathrm{cb}$, and the antecedent proposition to some possible worlds nearest to the current world, and that would and can' are truth-functionally relating these worlds to the worlds where the corsequent proposition holds. Since our main concern here is the interaction of the conditional interpretation and dake in $p$-dakesentences and dake-p-sentences, we will not go into the details of her analysis. It would suffice for us to express the relevant semantic contents in the following way. (In each case, $\rightarrow$ wouid, and $\rightarrow$ can express the modalized conditional operator in Kratzer's sense which irnplic. itly incorporates the conversational background, cb.)

(27) (Bill wears that new outfit)

$$
\rightarrow \text { would (Bill fools everyone) }
$$

(28) (John stands on a cliair)

$$
\rightarrow \text { can (John touches the ceiling) }
$$

Since with-phrases in Lnglish behave semantically as free adjuncts, we can get the conditional interpreta. tion for the English sentence (21) from the semantics of "possible" predicates. The semantic content can be expressed as in (29).

(29) (I use a bike) $\rightarrow$ can (I get there)

Assuming that de-phrases in Japanese behave semantically like free adjuncts in English, we can get the conditional interpretation for (14) fully from the semantics of "possible" predicates in a similar way. The semantic content would roughly be the sume as (29).

\subsection{Interaction of dake, de, and "Possi- ble" Contexts}

Now we have come to a place where we can resolve the problems about the difference in meaning between p-dake-sentences and dake-p-sentences. But to do so, first we have to take a further look into the nature of dake.

\subsubsection{Only as a Focusing Operator}

There has been a fair amount of work on the semantics of only. The basic semantic content of Japanese dake is presunably almost the same as English only.

Only is said to be a focusing operator[11]. This is because the truth condition of the sentences with only depends on so-called focus, as shown in (30). (Focus elements are written in capital letters.)

(30) a. John only introduced BII,I, to Sue $=$ John introduced only Bill to Sue

b. John only introduced Bill to SUF, = John introduced Bill to only Sue

liach of these sentences can be paraphrased as the sentences below them, which succinctly slow the difference in their truth conditions.

The traditional treatment for this fact is provided by formulating the semantic contents of only as a binary function which maps its focus element and scope element to something of the appropriate type. The logical form of these sentences can be written in the following way.

(31) a. only(Bill) $(\lambda x[$ Joln introduced $x$ to Sue] $)$

b. orly (Sue) $(\lambda x[$ John introduced Bill to $x])$

In these formulae, the first argument is the focus of only, and the second argument is its scope. When you supply the appropriate Intensional Logic translation to this function $o n l y$, the equivalent Intensional Logic expressions for them results, as shown in (32).

(32) a. $\forall x\left[\right.$ introduced $\left.\left(j^{\prime}, x, s^{\prime}\right) \rightarrow x=b^{\prime}\right]$

b. Vx[introduced $\left.\left(j^{\prime}, b^{\prime}, x\right) \rightarrow x=s^{\prime}\right]$

There are problems with this kind of naive approach. See Rooth[9] and von Stechow[11] for some criticisms and possible extensions. Ilere, however, we will simply assume that dake immediately follows its focus element.

\subsubsection{How Do They Interact in Semantics?}

How much of the difference in meaning between p-dakesentences and dake-p-sentences can we uccount for in the semantics? From the discussions we gave in the last section, we can obtain the conditional interpretation as their semantic contents.

A similar paraphrase for (1) will work. Here again, we can paraphrase the sentences into something like (33) withont causing any difference in interpretation.

(33) a. Soko-ni-wa zitensya-o tukatte dake there-LOC-TOP bike-ACC using only iku-koto-ga-dekiru. go-that-NOM-can (Only if (I) use a bike, (I) can get there.)

b. Soko-ni-wa zitensya dake o tukatte there-LOC-TOP bike only ACC using iku-koto-ga-dekiru. go-that-Nom-can (If (I) use only a bike, (I) can get there.) 
One possible way we can think of $(33, \mathrm{a})$ is that dake takes entire de phrase as its focus and takes the whole sentence as its scope. Then its logical form can be expressed as in (34).

(34) only((I use a bike $))\left(\lambda P\left(P \rightarrow_{c a n}(I\right.\right.$ get the re $\left.\left.)\right)\right)$

This can be translated into (35).

(35) $\forall P[[P \rightarrow$ ean $(I$ get there $)] \rightarrow P=($ I use a bike $)]$

In (34), dake takes its scope over the whole modalized conditional interpretation, restricting antecedent, condition for enabling me to get there only to "using a bike." This expresses the 'absolute restriction' meaning correctly. Since there is no such condition other than using a bike which enables me to get there, $I$ can't get there without a bike.

As for $(33, b)$, its focus is clearly the NP zitensya and its scope is the whole antecedent sentence. Thus we obtain (36), and its translation (37).

(36) only $(($ a bike $))(\lambda x($ I use $x)) \rightarrow$ can $(I$ g et there $)$

$$
\begin{aligned}
(\text { I use a bike) } \wedge \forall y[(I \text { use } y) & \rightarrow y=\text { bik e }] \\
& \rightarrow \text { can (I get there) }
\end{aligned}
$$

This time, dake takes scope over the de-phrase, whose semantic content is roughly "using a bike", and excludes any other means of transportation. The sernantic contents of the whole sentence would be something like, "if I use a bike and do not use any other means, I can get there." In other words, "it is sufficient for getting there that I use a bike and do not use any other means." This means that "it is not necessary for getting there that I use any means other than bike", which is exactly the 'necessity part' of the 'minimal restriction' meaning.

In this way, 'how' part of the first question we raised in 4.2 is solved. Although (B) is stating an assumption for dake-p-sentences in general, there is only one case where dake-p-sentences have the 'necessity' part of the 'minimal restriction' meaning, that is the case where such sentences have de-phrases and "possible" predicates. In such cases, these sentences can get conditional interpretations due to those two independent facts.

- De-phrases act like free adjuncts in English.

- Free adjuncts in "possible" context can receive conditional interpretations by the semantic nature of "possible" predicates.

\subsection{Conversational Implicature of Dake- sentences}

The remaining question is 'how' part of the second question in 4.2. We can restate the question as follows:

- How can the 'scalar' part of the 'minimal restriction' meaning be derived conversationally?

For this question, we observed in 4.2 that the 'scalar' part can be obtained when de-phrases and "possible" predicates interact. We saw in 5.1 that when de-phrases and "possible" predicates interact, we can get conditional interpretations. These facts suggest that the conditional interpretations of dake-de-sentences are somehow related to the 'scalar' implicature. Let us consider the original dake-de-sentence, again shown here.
(1) b. Soko-ni-wa zitensya dake de ik-eru. there-LOC-TOP bike only INST $i$ go-can ((I) can get there by bike alone.)

To get the 'scalar' implicature, we have to have some contextually salient scale. Often, certain specific linguistic expressions, such as (some, all), (bad, good), or numerals provides such scales, and 'generalized scalar implicature' is derived[6]. But in the particular case we are considering, the 'scalar' implicature is clearly contextually dependent, i.e., the scale have to be supplied by the context.

Basically, almost all utterances can have the 'scalar' implicature when they are placed in appropriate contexts. But how easily we can think of the appropriate scale varies. For $(1, b)$ and its conditional interpretation, we can think of such scale rather easily, i.e., the scale in relative easiness of various means for getting there. For example, bike is easier than walking, car is easier than bike, and airplane is easier than car, etc. But you can think of a context where this easiness changes the direction, i.e. bike is easier than car, and car is easier than airplane, etc. So the scale itself is totally context dependent. The important point is that this scale of easiness can be set based on the conditional interpretation of $(1, b)$. We have an inference pattern according to its conditional interpretation shown in (38).

$$
\frac{(\text { I use a bike }) \rightarrow \text { can (I get there) }}{(\text { I use a car }) \rightarrow \text { can (I get there) }}
$$

This means that the inference pattern of this kind can be made salient by the conditional interpretation of $(1, b)$. That's why the 'scalar' part of the 'minimal restriction' meaning comes with its 'necessity' part. These parts are independently derived from its conditional interpretation. The former is obtained semantically, the latter pragmatically.

\subsection{The Interpretive Procedure: The Whole Picture}

The preceding sections have given an overall picture of the procedure for the interpretations of sentences involving dake. We believe that our account is more effective and exhaustive than previous ones.

First, for $p$-dake-sentences, we get the 'absolute restriction' meaning in their semantics, taking the scope of dake over the whole sentence. For de-dake-sentences in particular, we get the 'absolute restriction' meaning of modalized conditional swhen they have "possible" predicates.

Second, for dake-p-sentences, we get the different semantic contents from their corresponding p-dakesentences when certain predicates are involved, due to 
the fact that dake takes scopes over their postpositional phrases. Then for dake-de-sentences with "possible" predicates, we get the conditional interpretation and the 'necessity' part of the 'minimal restriction' meaning in their semantics, in addition, the 'scalar' implicature can be obtained based on a context-dependent scale which is set by their conditional interpretations.

\section{Concluding Remarks}

Our main concern in this paper is the difference in interpretations between $p$-dake-sentences and dake-psentences, and its distribution with regard to particles with which dake interacts and properties of predicates involved. We started by reviewing some of the previous accounts of this phenomenon and pointed out their difficulties, summarizing our basic hypotheses which cover the semantics and the pragmatics for these sentences. By taking a closer look at related examples, we demonstrated that both de-phrases and "possible" predicates play crucial roles for the difference in available interpretations, suggesting interaction of semantic and pragmatic processes that would explain this difference. Finally, we have presented a general picture of how our interpretive procedure works for these sentences.

While most of our observations were restricted to Japanese sentences with dake, our approach can naturally be extended to the corresponding English sentences with only. We presume that the interpretive procedure we proposed in this paper is a universal one.

\section{Acknowledgements}

Michio Isoda brought our attention to the interaction of dake and de in Japanese. We would like to thank Peter Sells, Mark Gawron, and Chris Pinon for helpful comments. Special thanks are due to CSLI for providing an ideal environment for research and giving us opportunities to engage in stimulating and insightful discussions with researchers from various backgrounds.

\section{References}

[1] Grice, P.H.: 1975. Logic and Conversation. In P. Cole and J.L. Morgan (eds.), Syntax and Semantics, Vol.9: Speech Acts. Academic Press, 41-58.

[2] Karttunen, L., and Peters, S.: 1979. Conventional Implicature. In $\mathrm{C}-\mathrm{K}$. Oh and D. Dinneen (eds.), Syntax and Semantics, Vol.11. Academic Press, New York, 1-56.

[3] Kratzer, A.: 1979. Conditional Necessity and Possibility. In R. Bauerle et al. (eds.), Semantics from Different Point of View. Springer-Verlag, 117-147.

[4] Kratzer, A.: 1981. Blurred Conditionals. In W. Klein and W, Levelt (eds.), Crossing the Boundaries in Linguistics. Reidel, Dordrecht, 201-209.

[5] Kuno, S.: 1983. Shin Nihon Bunpoo Kenkyuu. Taishuukan-shoten.
[6] Levinson, S.C.: 1983. Pragmalics. Cambridge University Press.

[7] Lewis, D. : 1973. Counterfactuals. Harvard University Press.

[8] Morita, Y.: 1971. Dake, Bakari no Yoohoo. In Bulletin of The Institute of Language Teaching, Vol.10. Waseda University, 1-27.

[9] Rooth, M.E.: 1985. Associalion with Focus. PhD. Dissertation, UMass.

[10] Stump, G.: 1985. The Semantic Variability of $A b$. solute Constructions. Reidel, Dordrecht.

[11] von Stechow, A.: 1989. Focusing and Backgrounding Operators. In Fachgruppe Sprachwissenschaft der Universitat Konstanz, Arbeitspapier Nr.6. 